\title{
Techno-economic Analysis of Residential Thermal Flexibility for Demand Side Management
}

\author{
Jan Devriese \\ IDLab \\ imec-Ghent University \\ Ghent, Belgium \\ Didier Colle \\ IDLab \\ imec-Ghent University \\ Ghent, Belgium \\ didier.colle@ugent.be
}

javriese.devriese@ugent.be

\author{
Thibault Degrande \\ IDLab \\ imec-Ghent University \\ Ghent, Belgium \\ thibault.degrande@ugent.be
}

\author{
Mihail Mihaylov \\ AI Lab \\ Vrije Universiteit Brussel \\ Brussels, Belgium \\ mihail.mihaylov@vub.be
}

\author{
Sofie Verbrugge \\ IDLab \\ imec-Ghent University \\ Ghent, Belgium \\ sofie.verbrugge@ugent.be
}

\begin{abstract}
The continuing rise in solar and wind production leads to an increasing demand of flexibility to stabilize the electricity grid. Furthermore, we can assume a gradual but intensive rise in the use of electrical heatpumps for household spatial heating, for different reasons. Therefore, this paper investigates the feasibility and viability of entering the flexibility market by aggregating residential thermal loads. For this research, a dataset of 200 dwellings in the Netherlands, equipped with a heatpump and smart metering infrastructure, is analysed. By means of a greybox modeling approach, a thermal model and control framework have been set up for every house, in order to identify the load shift potential and the accompanying cost of providing flexibility for the houses. We find that thermal flexibility is asymmetric: downwards flexibility is, apart from much more dependent on outdoor temperature than upwards flexibility, strictly lower than upwards flexibility. The cost for downwards flexibility is strictly negative in terms of the prosumer. Concerning upwards flexibility, the cost is most of the time positive. Moreover, it can be concluded that there is a potentially viable business case for the flexibility aggregator.
\end{abstract}

Index Terms - demand side management, thermal modelling, flexibility markets, techno-economics

\section{INTRODUCTION}

On the $22^{\text {nd }}$ of April 2016, 196 countries signed the Paris Agreement [1], an engagement of every signing member to keep the global rise in temperature below $2^{\circ} \mathrm{C}$ and if possible below $1.5^{\circ} \mathrm{C}$. This engagement can only be accomplished by emitting less greenhouse gases. To this end we have to, amongst other things, alter the way we produce energy. Europe has set the goal to reach a $100 \%$ green European energy grid in 2050, in which a large part of electricity will be generated by solar and wind power, which are inherently intermittent and difficult to forecast. High forecasting errors will lead to an increase in imbalances in the electricity market.
In Belgium, the Transmission System Operator (TSO) Elia is responsible for maintaining the grid balance. It is important to notice that as of June 2003, the electricity market design in Belgium changed drastically due to partial liberalisation. This created new roles and actors engaging in the electricity market, buying and selling electricity. Such players can, for instance, apply demand side management (DSM). DSM, and in particular demand response (DR), can be established by actively controlling electrical loads [2]. Elia ensures the balance by imposing financial incentives on these market players. However, if this mechanism fails and the imbalance is too large, Elia can use ancillary services to restore these imbalances. These ancillary services are categorized by rate of response and named R1, R2 and R3 market. The tertiary reserve (R3) consists of contracts between Elia and the flexibility provider. The flexibility provider is a flexible generating capacity or large flexible consumer, which can alter its energy use on request of Elia. In the past, only CIPU (Coordination of the Injection of the Production Units) power plants, i.e. large generators with a generating capacity of over $25 \mathrm{MW}$ were able to participate in this product, but as from 2017, the minimal bid size was lowered to $1 \mathrm{MW}$ and was opened to nonCIPU (e.g. consumers or aggregated consumers) to enter this reserve product (e.g. R3-Flex). Hence, residential consumers can, via a flexibility aggregator, offer their flexible electrical loads to the tertiary reserve, in order to be used by Elia to balance the grid when needed. In the considered setup, the flexibility aggregator first submits an offer to TSO for a certain upcoming period of time, in which it specifies the delivery type and the volume and price. Next, if the offer is accepted, the TSO can, during the contracted period, request the flexibility aggregator 
to activate the specified volume of loads. An important enabler for the aggregator to satify such request is the rollout of the digital meter in Flanders, planned in July 2019. The flexibility aggregator is able to send a signal to the individual smart meters in the home, equipped with a NB-IoT communication module. In turn, the smart meter is able to interprete the signal and switch off/on (smart) electrical loads. Fig. 1 shows the setup of the ICT solution.

Apart from electricity production, the Flemish Energy Plan (2020-2030) intends to lower carbon emissions in spatial heating of residential buildings, by replacing old gas-powered heating systems with electrical heatpumps [3]. Substantial stimuli are foreseen towards this shift. Hence, a new electrical, flexible load will be present in Flemish households, giving rise to the opportunity for a flexibility provider to aggregate the flexibility of these heatpumps and to bid it on the R3 market. Hence, as the enablers and necessity come together, there is a clear need in the valorisation of thermal flexibility.

The main contributions of this paper are the validation of the business case for a flexibility aggregator to enter the R3-flex market of Elia and a data-driven analysis of the load shift potential of spatial heating using heat pumps, in the Netherlands. To the best of our knowledge, no research that valorises bidding on the R3-flex market of Elia using heatpump flexibility has been conducted.

The remainder of the paper unfolds as follows. In the following section a brief summary of related work will be given. Section 3 explains the methodology used in order to obtain the thermal model and control framework and explains the way in which the viability of entering the R3-flex market has been assessed. Section 4 shows the results for the load shift potential of the dwellings in the dataset and the results for the R3 market analysis. Section 5 covers the conclusions and future work.

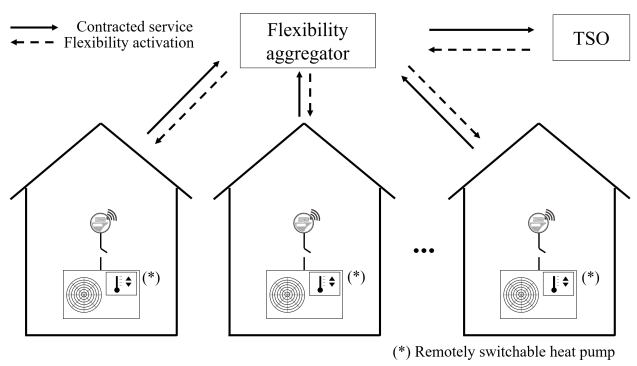

Fig. 1: Flexibility aggregator as new market actor

\section{BACKGROUND}

Numerous papers have been investigating the benefits and challenges of DSM in smart grids [4]-[6]. Demand side management, which is the collective name for demand response (DR) and energy efficiency [5], focuses on altering energy demand in order to deliver numerous ancillary services (load following, load curtailment, frequency regulation, tackle congestion problems, etc.).
However, the benefits of DR vary greatly depending on its use (type of ancillary service) and the specific outlook of the energy landscape [4]. It has been shown that the additional value of DSM initiatives is greatly dependent on the flexibility of the current power sources and the geographical zone where the initiatives are being deployed [4].

Generally speaking, there are two ways to quantify the flexibility of dwellings, being (1)top-down and (2) bottom-up. An example will be given for both approaches. In 2014, the project LINEAR quantified the amount of flexibility of five different household appliances by means of a field study for the case of Flanders. They installed smart appliances in a series of households and analysed the load shift potential of those appliances. As a result, they have found that the average maximum load shift potential is a $430 \mathrm{~W}$ increase at midnight (30 $\mathrm{min})$ and a $65 \mathrm{~W}$ decrease in the evening $(15 \mathrm{~min})$, per household [7]. However, the study lacks the thermal inertia of the dwellings as flexible load, due to the decoupling of the domestic heat water buffer (DHW) and spatial heating system. This gap is addressed in this paper. As an example of a top-down approach, Hao et al. has shown that thermostatically controlled loads (TCLs) have a great potential in usage for fast frequency regulation, due to their large population size and possibility to switch the loads simultaneously [8]. The model that they used for this is called the generalized battery model [9], which models the aggregation of thermal loads as a large battery in the electricity grid, acting as a buffer. In this paper, a bottom-up approach is used.

\section{APPROACH}

In order to be able to bid flexibility on the electricity market, the amount of flexibility available by heat pumps must be determined. A bottom-up modelling approach is used to caculate the individual flexibility of every house. Flexibility can be defined as the possible deviation in electricity consumption pattern of the heat pump.

\section{A. Control Framework}

The baseline electricity consumption or heating pattern of the heat pump is determined by the control framework. This is the algorithm that decides when the heat pump is working and at what frequency it is modulated, in order to minimize the cost of electricity, while not allowing the indoor temperature to drop below the setpoint temperature. The resulting heat plan is the Optimal Heat Plan (OHP). To determine this optimal heating plan for the individual houses, a thermal model of the houses is needed.

The thermal model simulates the thermal behaviour of the dwellings under consideration for the next 24 hours. The thermal model is at the core of this research, as the indoor temperature will provide the flexibility, which we are trying to quantify. It will be used in the control framework to perform the state transitions 
between two consecutive, discrete time periods. Three types of models can be used in order to predict indoor temperature: black, grey and white box models. In black box models, the system (building) is viewed in terms of inputs and outputs, without considering its internal workings. As there is not sufficient data and considering the flatness of the data, black box models cannot be used. White box models on the other hand require tremendous modelling effort per building, as white box modeling the thermal behaviour takes into account all system and building characteristics. As we aim to use this research in a more generic way, white box models cannot be used. Therefore, this research uses a grey box model approach, having a partial theoretical structure with data to complete the model. Grey box models are ideal for this research, as they are much more easy to apply on a large scale, but still keep the link with a thermodynamic model.

The dwellings are assumed to consist of one room, which has a uniform indoor temperature. Furthermore, it is assumed that the only heat influx originates from heat dissipated by the spatial heating system (SHS) powered by the heatpump and heat gains from solar radiation. Latter assumptions lead to a equivalent circuit that has both a resistor ( $\mathrm{R}$, thermal resistance) and a capacitor (C, thermal capacitance), a lumped first-order $\mathrm{RC}$ model. Using historic measurements on the indoor temperature of the houses, the electricity consumption of the heatpump and outdoor temperature, the parameters of this first-order lumped RC grey box model were fitted. An overview of the control framework approach is shown in Fig. 2.

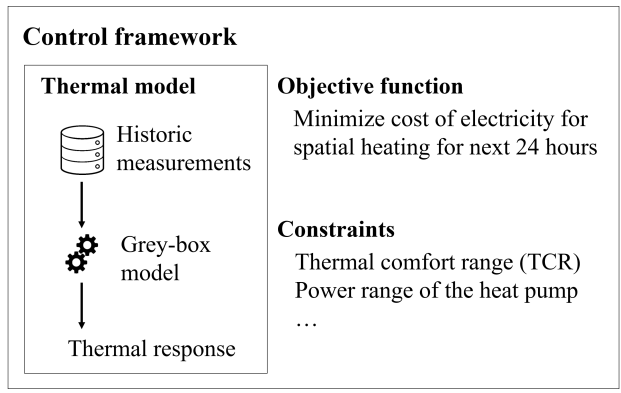

Fig. 2: Overview of the control framework

\section{B. Load shift potential of dwellings in dataset}

Based on the control framework, the load shift potential of the dwellings in the dataset can be calculated by quantifying the possible deviations of the OHP. These deviations are evidently limited by the maximum power of the heat pump and the thermal constraints imposed by the end-user. The first step is to identify the timeslot where the highest and lowest demand of the day occurred and this between 01/01/2018 and 01/05/2018. These extrema are the points in time where the highest need for load shift occurs. Only four months are considered because of computational limits. Moreover, heat pumps

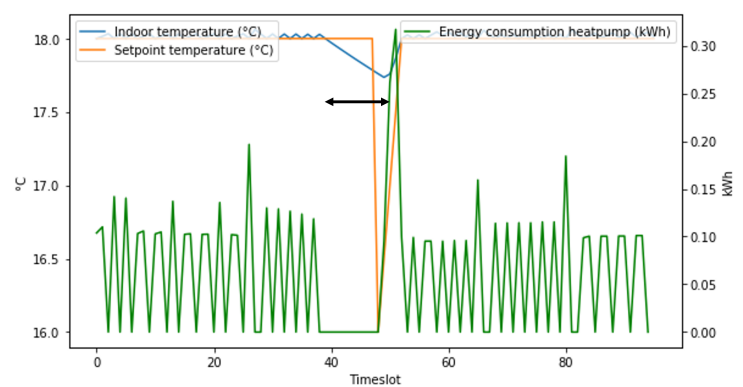

(a) Downwards flexibility

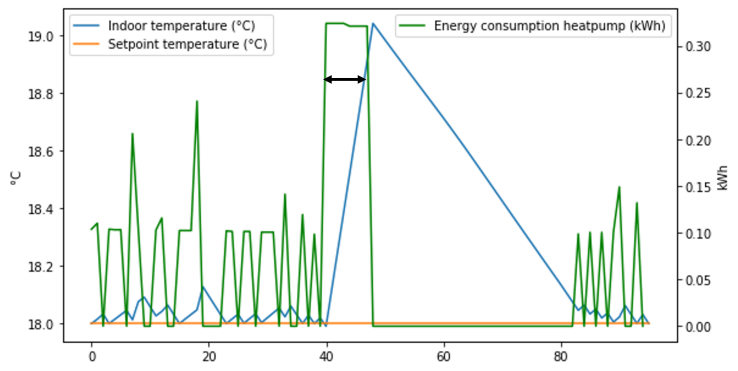

(b) Upwards flexibility

Fig. 3: Thermal flexibility potential for heat pump

will mainly generate heat in the colder months, so there is no value in analysing flexibility in the summer. The load shift potential is analysed by calculating the thermal flexibility of the dwellings during a time window around the peaks and lows. At the peak, downwards flexibility will be calculated and at the lows, upwards flexibility. This is done for a time window of $0.5,1$ and 2 hours and a thermal comfort range (TCR) of $+/-1^{\circ} \mathrm{C}$ and $2{ }^{\circ} \mathrm{C}$ relative to the setpoint temperature. Fig. 3 illustrates both upwards and downwards flexibility.

\section{Valorisation of flexibility}

The valorisation of the thermal flexibility in residential dwellings will be done by calculating the amount of houses needed to bid $1 \mathrm{MW}$ of capacity on the R3flex market [10]. This is a specific product of the third reserve of Elia and is currently only open to upwards bids. Amongst the requirements to bid on the R3-flex market are (1) guaranteed 100\% availability of full amount of contracted power, (2) maximum activation time of $2 \mathrm{~h}$, (3) maximum 8 activations per month, (4) minimum bid size of $1 \mathrm{MW}$. The contracts are settled on a monthly basis and the remuneration is $+/-€ 2500 / \mathrm{MW}$. The remuneration for the actual activation and delivering of the power is highly variable, so this is not taken into account. The main reasoning behind the calculation of the needed number of houses is as follows:

- 4 or 8 requests are generated on a random time, depending on the scenario;

- For all houses already aggregated, the upwards flexibility at the time of request is calculated; 
- If the total aggregated flexibility at a specific time of request is lower than $1 \mathrm{MW}$, a new house is generated, similar to the dwellings in the dataset;

- the series of setpoint temperatures for each house consists of an upwards flank in the morning and a downwards flank in the evening.

The valorisation is done by setting up a best, mid-range and worst case scenario. The best case scenario consist of zero activations, whereas the worst case consists of eight activations of maximum length. The mid-range scenario comprises four activation of random length.

\section{RESULTS AND DISCUSSION}

\section{A. Load shift potential}

1) Downwards flexibility: Fig. 4 displays the aggregated downwards flexibility for the houses (93) in the dataset from $1 / 1 / 2018$ to $1 / 5 / 2018$. Time interval of flexibility is 30 minutes (two slots) and the TCR is $4^{\circ} \mathrm{C}$. A first observation is that flexibility is not constant and varies between 0 and $-80 \mathrm{~kW}$, which is 0 to $0.86 \mathrm{~kW}$ per house. Note that this value is strongly dependent on outdoor temperature and the nominal power of the heat pump. These values are rather defensive, meaning that the houses are very well insulated (resulting in a relative high thermal capacitance) and the power of the heat pump is in the low end of the spectrum.

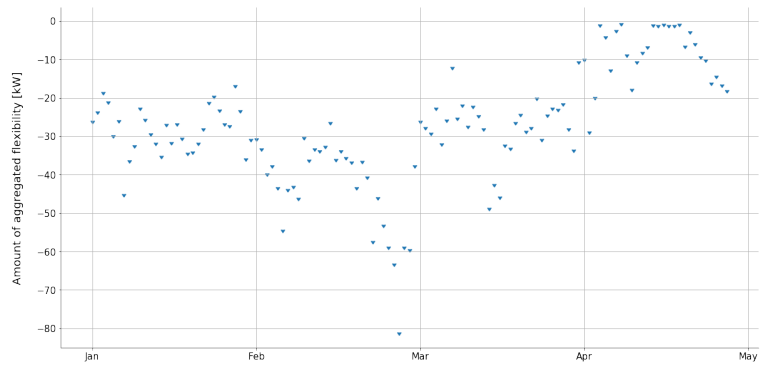

Fig. 4: Aggregated downwards flexibility

Fig. 5 shows the cost of providing downwards flexibility. Note that the cost is strictly negative, which is a logical consequence of lowering the setpoint temperature in the flexibility interval leading to an overall lower power consumption. The savings per house range from almost 0 to $€ 0.11$ per load shift of length 0.5 to 2 hours, depending on the request.

Important to notice is that the amount of downwards flexibility available is dependent on the outdoor temperature. This is because the upper bound of the flexibility available is the heat consumption of the baseline, which in turn goes down for rising outdoor temperature. Hence, flexibility approaches $0 \mathrm{kw}$ for higher temperatures.

2) Upwards flexibility: Fig. 6 displays the aggregated upwards flexibility. The conditions (TCR, Time of Flexibility (TOF)) are the same as in subsection IV-A1. Compared to downwards flexibility, upwards flexibility

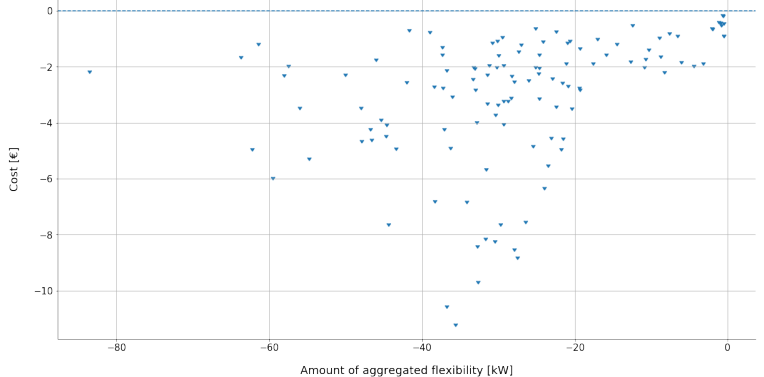

Fig. 5: Cost of aggregated downwards flexibility

is much higher and ranges between 68 to $106 \mathrm{~kW}$, except for two outliers. Note that the limiting factor here is not the TCR of $4^{\circ} \mathrm{C}$, but the power of the heat pump. Another difference is that upwards flexibility is much more constant than downwards flexibility. This is due to the absence of dependency of outdoor temperature. Furthermore, it has been found that the difference between a thermal comfort range of $2^{\circ} \mathrm{C}$ and $4^{\circ} \mathrm{C}$ is negligible for lower temperatures. However for higher outdoor temperatures, a larger TCR is needed to provide the same amount of flexibility.

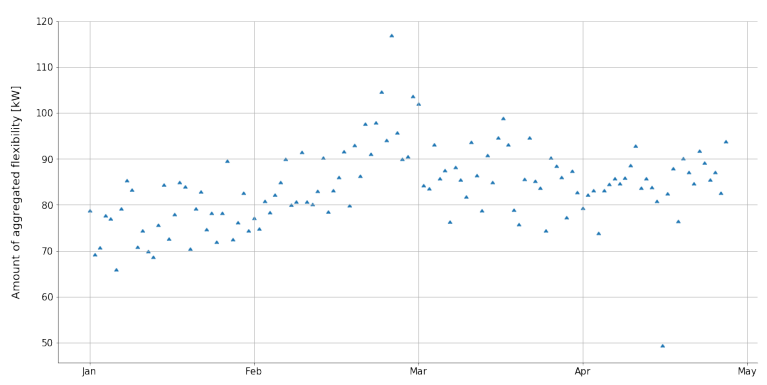

Fig. 6: Aggregated upwards flexibility

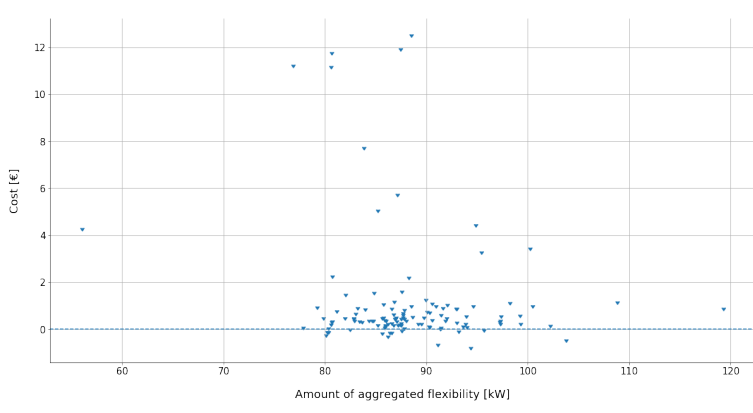

Fig. 7: Cost of aggregated upwards flexibility

Fig. 7 shows the cost of providing upwards flexibility. Note that the cost is not strictly positive, which is a result that was not expected. The cost per house ranges from $€-0.005$ to $€ 0.13$ per load shift of length 0.5 to 2 hours, depending on the request. The negative values can be the result of two different causes. The first being that the result is sub-optimal, due to a termination condition set in the optimizer, in order to fasten up the calculations. 
The second possibility is that when providing upwards flexibility, the house is heated at a time where the coefficient of performance (COP) of the heat pump is more favourable, as the COP is modelled with a temperature dependency and there is more freedom in the model introduced. This can lead to some small savings. The COP is the fraction of the effective heat that the heat pump is producing to the energy it consumes.

\section{B. Valorisation}

As explained in subsection III-C, the valorisation of entering the R3-flex market of Elia is done by considering a worst, best and mid-range scenario. The way these scenarios have been constructed is explained in section III-C. It is assumed that the increased electricity cost of the prosumers is completely reimbursed by the flexibility aggregator, but there are no additional remunerations for the prosumers. The experiment has been repeated for the first four months of 2018 and a TCR of 2, 4, 6 and $8{ }^{\circ} \mathrm{C}$. In Table II] the minimal amount of houses needed to fulfill the $1 \mathrm{MW}$ request is depicted for every setting. Notice that it allows to draw conclusions on the sensitivity of the results due to changes in the parameters TCR and outside temperature (later months are assumed to have a higher average outside temperature). As the R3 of Elia is currently only open to upwards bids, an extensive analysis was done on the impact of relevant parameters on upwards flexibility. For the effect of thermal comfort range, the general conclusion is that the higher the TCR, the less houses are needed to reach $1 \mathrm{MW}$. It can be seen from the table that for every month and for both worst and mid-range scenario, the number of houses decreases as the TCR increases. The reason for this is that the heat pump can work at higher loads or at maximum load for a longer time without reaching the upper thermal limit. The increase from $2^{\circ} \mathrm{C}$ to $4^{\circ} \mathrm{C}$ was found to be the most significant. For shorter TOFs, the influence of TCR is less pronounced. Except for situations with high outside temperatures, TCR has no significant influence on the total costs. However, increasing the TCR also increases the thermal discomfort of the prosumers.

Regarding outside temperature, it was found that a higher temperature leads to more houses needed, and more costs. This is due to the outdoor temperature leading to a more rapid rising indoor temperature. No interdependency between TCR and outside temperature was found.

A third parameter that was investigated is the time of flexibility (TOF). As the TOF is not incorporated in Table III the influence of the duration of the flexibility request on the results is summarized in Table I The table shows that an increase in TOF in general leads to a increase in the amount of houses needed to fulfill the request, together with the variability. Moreover, the total cost and cost per house increases significantly. The latter is explained by a higher thermal discomfort for longer activations. 1 Shorter requests lead to a negligible thermal discomfort and thus also negligible cost.

TABLE I: Influence of TOF on houses needed, total cost, cost per house and average discomfort temperature

\begin{tabular}{lllllllll} 
TOF [h] & \multicolumn{2}{c}{ Houses needed } & \multicolumn{2}{c}{ Total costs } & \multicolumn{2}{c}{ Cost/house } & \multicolumn{2}{c}{ Mean T_disc $\left[{ }^{\circ} \mathbf{C}\right.$ ] } \\
mean & std & mean & std & mean & std & mean & std \\
$\mathbf{0 . 2 5}$ & 314 & 42 & 7.77 & 4.50 & 0.02 & 0.01 & 0.10 & 0.09 \\
$\mathbf{0 . 7 5}$ & 314 & 54 & 25.87 & 9.18 & 0.08 & 0.03 & 0.29 & 0.08 \\
$\mathbf{1 . 5}$ & 496 & 207 & 150.06 & 162.19 & 0.29 & 0.29 & 0.85 & 0.46 \\
$\mathbf{2}$ & 468 & 226 & 160.42 & 104.13 & 0.40 & 0.29 & 0.81 & 0.43
\end{tabular}

Finally, no significant influence of the time of the day of the flexibility request on the number of houses or the costs was found. In Table II] the minimal amount of houses needed to fulfill the $1 \mathrm{MW}$ request is depicted for every setting.

It can be seen that for every month and for both worst and mid-range scenario, the number of houses decreases as the TCR increases. The reason for this is that the heat pump can work at higher loads or at maximum load for a longer time without reaching the upper thermal limit. However, increasing the TCR also increases the thermal discomfort of the prosumers. Next, for warmer months more houses are needed. This is due to the outdoor temperature being higher, leading to a more rapid rising indoor temperature.

TABLE II: Minimal number of houses needed per scenario and setting

\begin{tabular}{lllll} 
& & \multicolumn{3}{c}{ Minimal houses needed } \\
\hline TCR & Month & Worst case & Mid-range & Best case \\
\hline $\mathbf{2}$ & $\mathbf{1}$ & 1049 & 869 & 0 \\
& $\mathbf{2}$ & 924 & 728 & 0 \\
& $\mathbf{3}$ & 1078 & 736 & 0 \\
& $\mathbf{4}$ & 1319 & 1080 & 0 \\
\hline $\mathbf{4}$ & $\mathbf{1}$ & 574 & 459 & 0 \\
& $\mathbf{2}$ & 503 & 407 & 0 \\
& $\mathbf{3}$ & 568 & 387 & 0 \\
& $\mathbf{4}$ & 695 & 598 & 0 \\
\hline $\mathbf{6}$ & $\mathbf{1}$ & 451 & 378 & 0 \\
& $\mathbf{2}$ & 396 & 346 & 0 \\
& $\mathbf{3}$ & 442 & 322 & 0 \\
& $\mathbf{4}$ & 522 & 488 & 0 \\
\hline $\mathbf{8}$ & $\mathbf{1}$ & 402 & 356 & 0 \\
& $\mathbf{2}$ & 347 & 327 & 0 \\
& $\mathbf{3}$ & 396 & 304 & 0 \\
& $\mathbf{4}$ & 463 & 457 & 0 \\
\hline
\end{tabular}

Regarding revenue, the results of each scenario and the weighted total revenue can be seen in Table III In the weighted total, results of each scenario are combined using the PERT technique [11]. From Table III] it can be seen that for the worst and mid-range scenario, the revenue decreases month by month. This is due to higher outdoor temperatures, leading to more thermal discomfort and thus more electricity consumption compared to the optimal heat plan. Furthermore, the worst case scenario has severely decreasing revenues for the fourth month if the TCR becomes higher. Again, this is a result of higher thermal discomfort. Finally, the table

\footnotetext{
${ }^{1}$ As said before, higher thermal discomfort is a result of an overall daily increase in power consumption by the heat pump, thus resulting in a higher cost.
} 
shows that only in two cases of the worst case the revenue of the flexibility aggregator is negative. From these results one could conclude that bidding $1 \mathrm{MW}$ of flexibility on the R3-flex market of Elia is viable. However, this business case has major shortcomings as it does not take into account the costs of providing the flexibility (e.g. needed equipment at the prosumers to enable two-way communication, implementation costs). In-depth assessement of these costs should be carried out, as well as who would have to bear this costs. Furthermore, prosumers might need an incentive to participate in such aggregation programs, or be compensated for their thermal discomfort, which would also negatively affect the business case of the flexibility aggregator. A last point of attention is that the contracts are bid on a monthly basis and penalties for not delivering are in place.

TABLE III: Summary of valorisation scenarios

\begin{tabular}{lllll|l}
\hline & & \multicolumn{4}{c}{ Revenue $[€]$} \\
\hline $\mathbf{2}$ & Month & Worst case & Mid-range & Best case & Weighted total \\
\hline & $\mathbf{1}$ & 1695 & 2339 & 2500 & 2259 \\
& $\mathbf{2}$ & 1650 & 2303 & 2500 & 2227 \\
& $\mathbf{3}$ & 1900 & 2304 & 2500 & 2269 \\
& $\mathbf{4}$ & 850 & 1809 & 2500 & 1764 \\
\hline $\mathbf{4}$ & $\mathbf{1}$ & 1507 & 2332 & 2500 & 2223 \\
& $\mathbf{2}$ & 1619 & 2306 & 2500 & 2224 \\
& $\mathbf{3}$ & 1728 & 2264 & 2500 & 2214 \\
& $\mathbf{4}$ & 271 & 1714 & 2500 & 1605 \\
\hline $\mathbf{6}$ & $\mathbf{1}$ & 1379 & 2329 & 2500 & 2199 \\
& $\mathbf{2}$ & 1598 & 2308 & 2500 & 2222 \\
& $\mathbf{3}$ & 1612 & 2268 & 2500 & 2197 \\
& $\mathbf{4}$ & -100 & 1655 & 2500 & 1503 \\
\hline $\mathbf{8}$ & $\mathbf{1}$ & 1311 & 2337 & 2500 & 2193 \\
& $\mathbf{2}$ & 1628 & 2310 & 2500 & 2228 \\
& $\mathbf{3}$ & 1484 & 2278 & 2500 & 2183 \\
& $\mathbf{4}$ & -331 & 1631 & 2500 & 1449 \\
\hline
\end{tabular}

\section{CONCLUSION}

\section{A. Load shift potential}

It can be concluded that thermal residential flexibility is asymmetric. Downwards flexibility is strictly lower than upwards flexibility and downwards flex is much more dependent on outdoor temperature. The amount of flexibility is in both cases very dependent on the nominal power of the installed heatpump. For larger nominal power the thermal comfort range becomes a limiting factor for flexibility. The cost of downwards flexibility is strictly negative, but is too low to be an incentive for prosumers to take part in demand response initiatives. The cost of upwards flexibility is not strictly positive but is fairly low.

\section{B. Valorisation of thermal flexibility}

The amount of houses needed to bid 1 MW power capacity on the R3-flex market is highly variable and ranges between 304 and 1319 houses and comes with a cost of $€ 0.23$ to $€ 7.26$ per house per request. Taken into account that the houses in the dataset are well insulated and the model of the heat pump has a low power, the business case for entering R3-flex with only thermal flexibility as a resource is expected to be viable.
However, this statement should be treated carefully, as the contracts are bid on a monthly basis and penalties for not delivering are in place. Furthermore, the business case omits costs aspects that could negatively affect the business case, such as additional implementation costs and willingness to participate. For high outdoor temperatures, the cost of providing flexibility is higher than at lower temperatures, so contracts should be made for colder months.

\section{Further work}

Improvements could be made to the thermal model in order to make it less biased. The point of focus should be improving the solar heat gain coefficients by calculating them taking more factors into account. Further improvements to be made in the control framework is taking modulation and supply heat water into account in modeling the COP of the heatpump. Also, a controlling algorithm should be constructed from the aggregator point of view, which should prioritize houses with the lowest thermal discomfort. Further work in the valorisation is making the group of houses more heterogeneous to give a more realistic view. As discussed, an in-depth assessment of all the costs of providing the flexibility should be carried out. Further research could also look into the potential of thermal flexibility in general or in the total of Belgium, and the potential of thermal flexibility in addressing the balancing issue as a whole.

\section{REFERENCES}

[1] United Nations, "Paris Agreement," 2015.

[2] G. Strbac, "Demand side management: Benefits and challenges," Energy Policy, vol. 36, no. 12, pp. 4419 - 4426, 2008, foresight Sustainable Energy Management and the Built Environment Project.

[3] Flemish Government, "Ontwerp Vlaams Energieplan 2021-2030," 2018.

[4] G. Strbac, "Demand side management: Benefits and challenges," Energy Policy, vol. 36, no. 12, pp. 4419 - 4426, 2008, foresight Sustainable Energy Management and the Built Environment Project.

[5] M. Behrangrad, "A review of demand side management business models in the electricity market," Renewable and Sustainable Energy Reviews, vol. 47, pp. 270 - 283, 2015.

[6] N. G. Paterakis, O. Erdinç, and J. P. Catalão, "An overview of demand response: Key-elements and international experience," Renewable and Sustainable Energy Reviews, vol. 69, pp. 871 891, 2017.

[7] R. D'hulst, W. Labeeuw, B. Beusen, S. Claessens, G. Deconinck, and K. Vanthournout, "Demand response flexibility and flexibility potential of residential smart appliances: Experiences from large pilot test in Belgium," Applied Energy, vol. 155, pp. 79-90, oct 2015.

[8] H. Hao, B. M. Sanandaji, K. Poolla, and T. L. Vincent, "Potentials and economics of residential thermal loads providing regulation reserve," International Journal of Electrical Power \& Energy Systems, 2015.

[9] H. Hao, B. M. Sanandaji, K. Poolla, and T. L. Vincent, "A generalized battery model of a collection of thermostatically controlled loads for providing ancillary service," pp. 551-558, Oct 2013.

[10] Elia, "General framework for tertiary control service by non-cipu technical units," Jan. 2019.

[11] "Use pert technique for more accurate estimates." [Online]. Available: https://www.techrepublic.com/blog/it-consultant/usepert-technique-for-more-accurate-estimates/ 